\title{
1997 Toxic Chemical Release Inventory Emergency Planning and Community Right-to-Know Act, Section 313
}

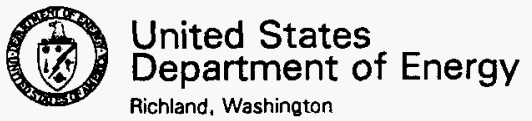


TRADEMARK DISCLAMER

Reference herein to eny specific commerciel product, process, or sonvice by trede name, tredemark, manufecturer, or otherwise, does not necessarily constitute or imply its endorsement, recommendation, or favoring by the United States Government or any sgency thereof or its contractors or subeontractors.

This report has been reproduced from the best aveilable copy. Available in paper copy and microfiche.

Available to the U.S. Department of Energy and its contractors from

Office of Scientific and Technical Information

P.O. Box 62

Oak Ridge, TN 37831

(615) 576-8401

Available to the public from the U.S. Department of Commerce National Technical Information Service

5285 Port Royal Road

Springfield, VA 22161

(703) $487-4650$

Printed in the Unitad States of Amarice

DISCLM-5.CHP (8-91) 


\section{Toxic Chemical Release Inventory Emergency Planning and Community Right-to-Know Act, Section 313}

Date Published

June 1998 


\section{INFORMATION CLEARANCE FORM}

\begin{tabular}{|c|c|c|}
\hline \multicolumn{2}{|c|}{ A. Information Category } & B. Document Number DOE/RL-98-39 \\
\hline Abstract & $\square$ Journal Article & C. Title \\
\hline Summary & $\square$ Internet & 1997 TOXIC CHEMICAL RELEASE INVENTORY \\
\hline Visual Aid & Software & \\
\hline Fulj Paper & X Report & \\
\hline Other & & et \\
\hline
\end{tabular}

E. Required Information

1. Is document potentially Classified? $\mathrm{X}$ No $\square$ Yes (MANDATORY)

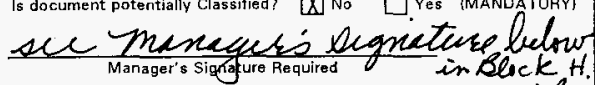
4. Does information Contain the Following: (MANDATORY)

a. New or Novel (Patentable) Subject Matter? $\square$ No $\square$ Yes If "Ves". Disclosure No:

If Yes $\square$ No $\square$ Yes Classified

b. Information Received in Confidence, Such as Proprietary and/or Inventions?

X. No $\square$ Ves If "Yes", Affix Appropriate Legends/Notices. ADC Signature Required $\square$ No $\square$ Y Yes

2. Internal Review Required? If Yes, Document Signatures Below Copyrights? XNo $\square$ Yes If "Yes", Attach Permission.

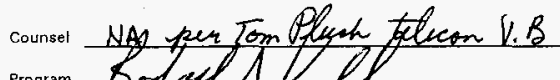
d. Trademarks? $X$ No $\square$ Yes If "Yes", Identify in Document. Natel

3. References in the Information are Applied Technology

Export Controlled Information X $]_{\text {no }} \square$ Yes 5. Is Information requiring submission to OSTI? $\square$ No $\square$ Yes If Yes $U C-630$ and $B \& R-E W 3110010$

Release Level? $\mathrm{X}$ Public $\square$ Limited $X]_{\text {no }} \square$ res 7. charge code RlA15 HAN 72300

\section{$\dot{F}$. Complete for a Joumal Article}

1. Title of Journal NA

\section{G. Complete for a Presentation}

1. Title for Conference or Meeting NA

2. Group Sponsoring

3. Date of Conference

5. Will Information be Published in Proceedings? $\square$ No $\square$ Yes

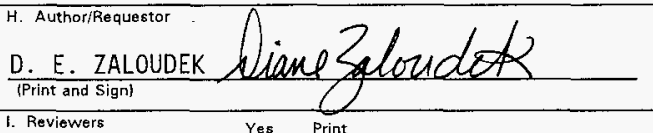

1. Reviewers

General Counsel

Office of External Affairs

DOE-RL

$\otimes$

R.N. Krekel

Other

Other

1. If Information Includes Sensitive Information and is not to be released to the Public indicate category below.

$\square$ Applied Technologr

$\square$ Personal/Private

$\square$ Proprietary

$\square$ Business-Sensitive

$\square$ Predecisional

$\square$ uCNI

K. If Additional Comments, Please Attach Separate Sheet
4. City/State

6. Will Material be Handed Out? $\square$ No $\square$ Yes

A. G. WEINER ifeN WuLiL (Print and Sign)

Signature

Public Y/N Ilf N, complete J)

$Y / N$

$Y / N$

Q $N$

$Y / \mathrm{N}$

$Y / N$ 


\section{RELEASE AUTHORIZATION}

Document Number: $\quad$ DOE /RL-98-39

Document Title: $\quad 1997$ Toxic Chemical Release Inventory $\quad$ Emergency Planning and Community Right-To-Know Act, Section 313

This document, reviewed in accordance with DOE Order 1430.1D, "Scientific and Technical Information Management," and DOE G 1430.1D-1, "Guide to the Management of Scientific and Technical Information," does not contain classified or sensitive unclassified information and is:

\section{APPROVED FOR PUBLIC RELEASE}

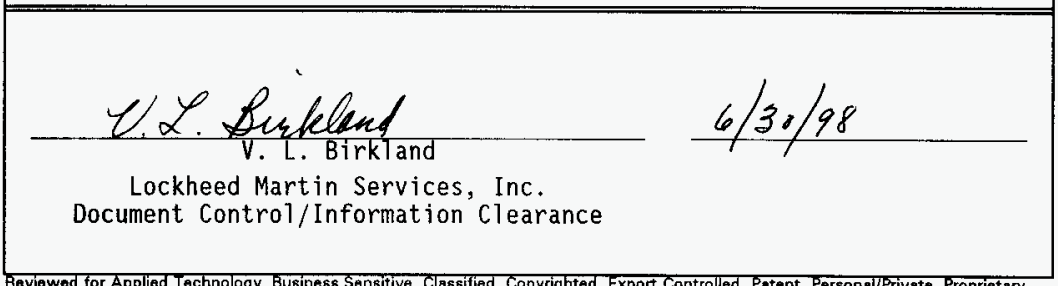

Roviewed for Applied Technology. Business Sensitive. Classified, Copyrighted, Export Controlled, Patent, Personal/Private, Proprietary.

Protected CRADA, Trademark, Unclassified Controlled Nuclear Information.

Trademark Disclaimer. Reference herein to any specific commercial product, process, or service by trade name, trademark, manufacturer, or otherwise, does not necessarily constitute or imply its endorsement, recommendation, or favoring by the United States Government or any agency thereof or its contractors or subcontractors. The viows and opinions of authors expressed herein do not necessarily state or reflect those of the United Stetes Government or any agency thereof. This report has been reproduced from the best available copy.

Printed in the United States of America.

Available to the U.S. Department of Energy and its contractors from the U.S. Department of Energy Office of Scientific and Technical Information, P.O. Box 62. Oak Ridge, TN 37831: Telephone: 423/576-8401.

Available to the public from the U.S. Department of Commerce National Technical Information Service, 5285 Port Royal Road, Springfield, VA 22161; Tolephone: 703/487-4650. 


\title{
1997 HANFORD SITE TOXIC CHEMICAL RELEASE INVENTORY DOE/RL-98-39
}

\section{CONTENTS}

\author{
Preface \\ (consisting of 1 page)
}

Memorandum: Delegation of Signature Authority for the Toxic Chemical Release Inventory (consiving of I page)

\section{Reporting Form A for Chlorine (comsinting of 2 pages)}

\author{
Reporting Form R for Phosphoric Acid \\ (comsisting of 5 pages)
}




\section{HANFORD SITE TOXIC CHEMICAL RELEASE INVENTORY}

DOE/RL-98-39

\section{PREFACE}

The Hanford Site covers approximately 1,450 square kilometers (560 square miles) of land that is owned by the U.S. Government and managed by the U.S. Department of Energy, Richland Operations Office (DOE-RL). The Hanford Site is located northwest of the city of Richland, Washington. The city of Richland adjoins the southeastern portion of the Hanford Site boundary and is the nearest population center.

The primary mission of the Hanford Site includes waste management, environmental restoration, and research and technology development. Toxic chemical activities managed by DOE-RL and its contractors in support of this mission were considered during preparation of the Toxic Chemical Release Inventory. This report does not include activities conducted by others on lands covered by leases, use permits, easements, and other agreements whereby land is used by parties other than DOE-RL. For example, this report does not include activities conducted by US Ecology, the Bonneville Power Administration, the National Science Foundation (the Laser Interferometer Gravitational-Wave Observatory), the Washington Public Power Supply System, Johnson Controls, Inc. (boilers operated for steam production), R. H. Smith Company (gas stations), or similar activities conducted on leased properties not under the management of DOE-RL.

Two listed toxic chemicals were used at the Hanford Site above established activity thresholds: phosphoric acid and chlorine. Because total combined quantities of chlorine released, disposed, treated, recovered through recycle operations, combusted for energy recovery, and transferred to off-site locations for the purpose of recycle, energy recovery, treatment, and/or disposal, amounted to less than 500 pounds, the Hanford Site qualified for the alternate one million pound threshold for chlorine. Accordingly, this Toxic Chemical Release Inventory includes a Form A for chlorine, and a Form R for phosphoric acid. 


\author{
QRTE: DEC 17992 \\ RETT OF: EAP:EBD 93-RPA-072 \\ SUSAET: DELEGATION OF SIGNATURE AUTHORITY FOR THE TOXIC CHEMICAL RELEASE INVENTORY \\ (SARA 313 TRI REPORT)
}

To: James D. Bauer, Acting Program Manager

Office of Environmental Assurance,

Permits, and Policy

I hereby delegate signature authority for the Toxic Chenica] Release Inventory (SARA 313 TRI Report) to the Program Manager, Ofifice of Environmental Assurance, Permits, and Policy.

Pursuant to 40 CFR Section 372.85, Toxic Chemical Reporting Forn and Instructions, part (b) (2), signature authority may be delgated by the manager to a senior management ofijcial. This memorandum constitutes formal delegation of such authority to the Progran Manager, office of Environmental Assurance, Permits, and Policy.

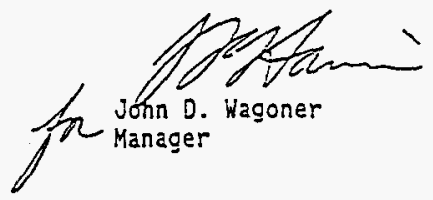




\section{EPA}

United States

Environmental Protection Agency
FORM R

Section 313 of the Emergency Planning and Community Right-to-Know Act of 1986,

also known as Title III of the Superfund Amendments and Reauthorization Act

TOXIC CHEMICAL RELEASE
WHERE TO SEND COMPLETED FORMS: 1 EPCRA Reporting Center P. O BOX 3348

Merrifield, VA $22116-3348$

ATTN: TOX!C CHEMICAL RELEASE INVENTORY
2. APPROPRIATE STATE OFFICE (See instructions in Appendix F)

Important: See instructions to determine when "Not Applicable (NA)" boxes should be checked.

\section{PART I. FACILITY IDENTIFICATION INFORMATION \\ SECTION 1.REPORTING YEAR 1997 \\ SECTION 2. TRADE SECRET INFORMATION}

\begin{tabular}{|c|c|c|c|c|c|c|c|}
\hline \multirow{2}{*}{2.1} & \multicolumn{3}{|c|}{ Are you claiming the toxic chemical identified on page 3 trade secret? } & \multirow{2}{*}{2.2} & \multirow{2}{*}{ Is this copy } & \multirow{2}{*}{ Sanitized } & \multirow{2}{*}{ Unsanitized } \\
\hline & $\begin{array}{l}\text { Yes. (Answer question } 2.2 \\
\text { Attach substantiation forms) }\end{array}$ & $x$ & $\begin{array}{l}\text { No (Do not answer 2.2: } \\
\text { Go to Section 3) }\end{array}$ & & & & \\
\hline
\end{tabular}

\section{SECTION 3. CERTIFICATION (Important: Read and sign after completing all form sections.)}

I hereby certify that I have reviewed the attached documents and that, to the best of my knowiedge and belief, the submitted information is true and complete and that the amounts and values in this report are accurate based on reasonable estimates using data available to the preparers of this report.

Name and official title of owner/operator or senior management official GEORGEH. SANDERS ACTING FOR ENVIRONMENTAL ASSURANCE. PERMITS DIRECTOR OF AND POLICY

\section{SECTION 4. FACILITY IDENTIFICATION}

4.1 Facility or Establishment Name U.S. DOE HANFORD SITE

\section{Street}

825 JADWIN AVENUE

\section{City/County/State/Zip Code}

RICHLAND

BENTON

WA

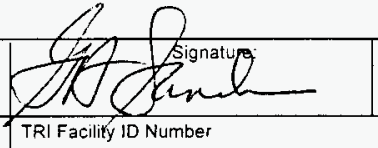
$06 / 30 / 98$

\begin{tabular}{|c|l|l|l|l|}
\hline 4.2 & $\begin{array}{l}\text { This report contains information for: } \\
\text { (Important check a or b; check c if applicable }\end{array}$ & $\begin{array}{l}\text { An entire } \\
\text { facility }\end{array}$ \\
\hline 4.3 & Technical Contact Name & RANDALL N. KREKEL & KAREN K. RANDOLPH & T. \\
\hline 4.4 & Public Contact Name & KA & \\
\hline
\end{tabular}

Facility or Establishment Name or Mailing Address(if different from street address)

\begin{tabular}{|c|c|c|c|c|c|c|c|c|}
\hline 4.5 & \multicolumn{2}{|c|}{ sIC Code (s) (4 digits) } & a. 9999 & 9511 & c. NA & d. & e. & f. \\
\hline \multirow{2}{*}{4.6} & \multirow{2}{*}{ Latitude } & Degrees & Minutes & Seconds & \multirow{2}{*}{ Longitude } & Degrees & Minutes & Seconds \\
\hline & & 046 & 30 & 00 & & 119 & 30 & 00 \\
\hline
\end{tabular}

\begin{tabular}{|l|l|c|l|l|l|l|l|l}
\hline 4.7 & $\begin{array}{l}\text { Dun \& Bradstreet } \\
\text { Number(s) (9 digits) }\end{array}$ & 4.8 & $\begin{array}{l}\text { EPA identification Number } \\
\text { (RCRA I.D. No.) (12 characters) }\end{array}$ & 4.9 & $\begin{array}{l}\text { Facility NPDES Permit } \\
\text { Number(s) (9 characters) }\end{array}$ & $\begin{array}{l}\text { 4.10 } \\
\text { (Rnderground Injection Well Code } \\
\text { (UIC) I.D. Number(s) (12 digits) }\end{array}$ \\
\hline 034456186 & W. WA7890008967 & WA0025917 & a. NA
\end{tabular}
b. NA
b. NA
b. WARO0000F

b.

\section{SECTION 5. PARENT COMPANY INFORMATION}

\begin{tabular}{|l|l|l|l|l|}
\hline 5.1 & Name of Parent Company & $\square$ & \multicolumn{2}{|l|}{ U.S DEPARTMENT OF ENERGY } \\
\hline 5.2 & Parent Company's Dun \& Bradstreet Number & $x$ & NA & \\
\hline
\end{tabular}




\section{SECTION 4. FACILITY IDENTIFICATION (CONTINUED)}

\begin{tabular}{|c|c|c|c|c|c|c|c|c|c|}
\hline 4.4 & \multicolumn{9}{|c|}{ Intentionally left blank } \\
\hline 4.5 & $\begin{array}{l}\text { SIC Code } \\
\text { (4-digit) }\end{array}$ & a. 9999 & b. 9511 & c. NA & d. & & $e$. & & f. \\
\hline \multirow{3}{*}{4.6} & \multirow{3}{*}{$\begin{array}{l}\text { Latitude and } \\
\text { Longitude }\end{array}$} & \multicolumn{3}{|c|}{ Latitude } & & \multicolumn{4}{|c|}{ Longitude } \\
\hline & & Degrees & Minutes & Seconds & & Degrees & & Minutes & Seconds \\
\hline & & 046 & 30 & 00 & & 119 & & 30 & 00 \\
\hline
\end{tabular}

\subsection{Dun and Bradstreet Number(s) (9 digits)}

4.8 EPA Identification Number(s) (RCRA I.D. No.) (12 characters)

a. 034456186

b. NA

a. WA7890008967

b. NA

4.9 Facility NPDES Permit Number(s)

(9 characters)

a. WA0025917

b. WARO0000F

4.10 Underground Injection Well Code (UIC) I.D. Number(s)

(12 digits)

a. NA

b.

\section{SECTION 5. PARENT COMPANY INFORMATION}

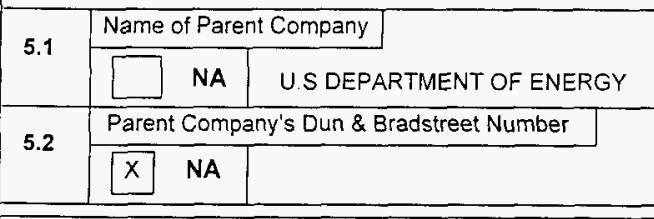

\section{PART II. CHEMICAL IDENTIFICATION}

\section{SECTION 1. TOXIC CHEMICAL IDENTITY}

\begin{tabular}{|c|c|}
\hline \multirow[t]{2}{*}{1.1} & CAS Number (important: Enter only one number exactly as it appears on the Section 313 fist. Enter category code if reporting a chemical category.) \\
\hline & 007782505 \\
\hline \multirow[t]{2}{*}{1.2} & Toxic Chemical or Chemical Category Name (lmportant; Enter only one name exactly as it appears on the Section $3 i 3$ list.) \\
\hline & CHLORINE \\
\hline \multirow{2}{*}{1.3} & Generic Chemical Name (lmportant: Complete only if Part I, Section 2.1 is checked "yes". Generic Name must be structurally descriptive). \\
\hline & NA \\
\hline
\end{tabular}

\section{SECTION 2. MIXTURE COMPONENT IDENTITY}


EPA United States

EPA Environmental Protection Agency

\section{TOXIC CHEMICAL RELEASE INVENTORY} FORM A
WHERE TO SEND THIS STATEMENT:
1. EPCRA Reporting Center

P.O. Box 3348

Merrifield, VA 22116-3348

ATTN: TOXIC CHEMICAL RELEASE INVENTORY
2. APPROPRIATE STATE OFFICE

(See instructions in Appendix $F$ )
Enter "X" here if

this is a revision

\section{PART I. FACILITY IDENTIFICATION INFORMATION}

\begin{tabular}{|c|c|c|c|c|}
\hline \multirow{3}{*}{$\begin{array}{l}\text { SECTION } 1 . \\
\text { REPORTING } \\
\text { YEAR }\end{array}$} & \multicolumn{4}{|c|}{ SECTION 2. TRADE SECRET INFORMATION } \\
\hline & \multirow[b]{2}{*}{2.1} & \multicolumn{3}{|c|}{ Are you claiming the toxic chemical identified on page 2 trade secret? } \\
\hline & & $\begin{array}{l}\text { Yes. (Answer questi } \\
\text { Attach substantiatior }\end{array}$ & $\bar{x}$ & $\begin{array}{l}\text { No: Do not answer } 2.2 \text {; continue } \\
\text { with Section } 3 \text {. }\end{array}$ \\
\hline 1997 & 2.2 & If yes in 2.1 , is this copy: & Sanitized & Unsanitized \\
\hline
\end{tabular}

\section{SECTION 3. CERTIFICATION (Important: Please read and sign after completing the statement.)}

I hereby certify that to the best of my knowledge and belief, for the toxic chemical listed in this statement, the annual reportable amount, as defined in 40 CFR 372.27(a), did not exceed 500 pounds for this reporting year and that the chemical was manufactured, processed, or otherwise used in an amount not exceeding 1 million pounds during this reporting year. Name and official title of owner/operator or senior management official

\begin{tabular}{ll}
\hline GEORGE H. SANDERS, ACTING FGR D)RECTOR OF ENVIRONMENTAL ASSURANCE. PERIMITS. AND POLICY \\
\hline
\end{tabular} Signature

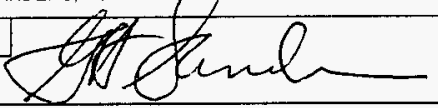

\section{SECTION 4. FACILITY IDENTIFICATION}

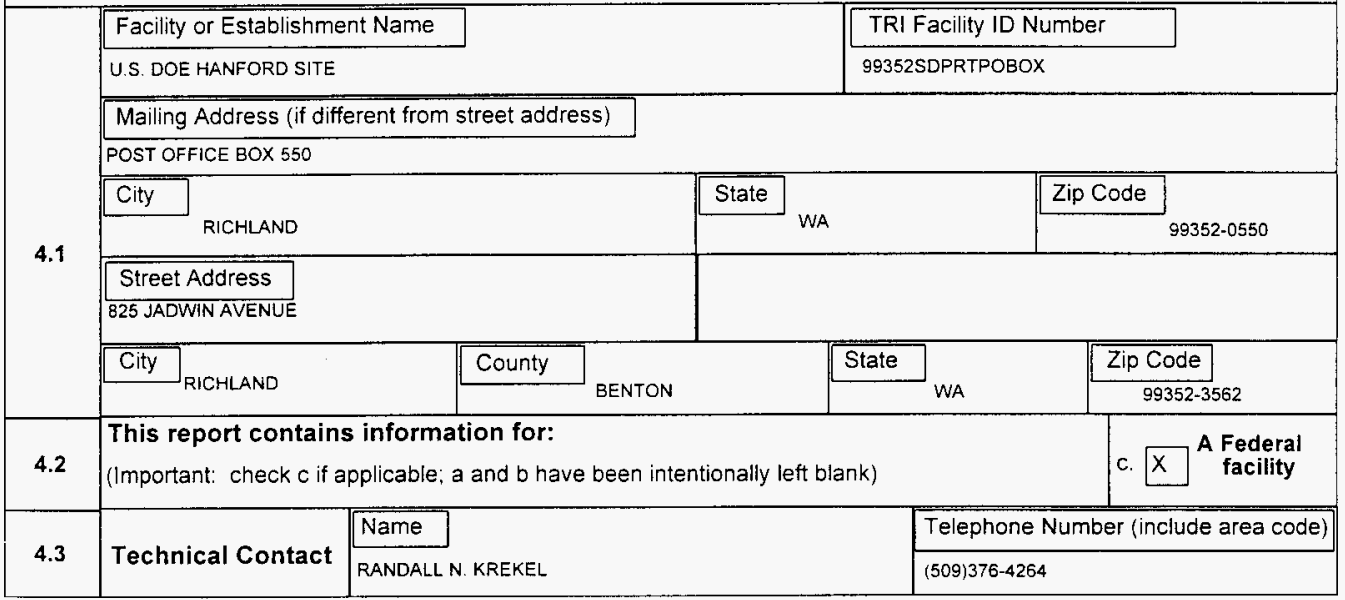




\section{EPA FORM R \\ PART II. CHEMICAL-SPECIFIC INFORMATION}

TRI FACILITY ID NUMBER

99352SDPRTPOBOX

Toxic Chemical, Category or Generic Name

PHOSPHORIC ACID

\section{SECTION 1. TOXIC CHEMICAL IDENTITY}

(Important: DO NOT complete this section if you complete Section 2 below.)

1.1 CAS Number (Important: Enter only one number exactly as it appears on the Section 313 list. Enter category code if teporting a chemical categury.) 007664382

1.2 Toxic Chemical or Chemical Category Name :Important: Enter only one name exactly as it appears on the Section 313 list.) PHOSPHORIC ACID

1.3 Generic Chemical Name (Important: Completionly if Part 1. Section 2.1 is checked "yes". Generic Name must be structurally descriptive) NA

\section{SECTION 2. MIXTURE COMPONENT IDENTITY limportant: DO NOT complete this section if you}

2.1 Generic Chemical Name Provided by Supplier (Important: Maximum of 70 characters, including numbers, lettess, spaces, and punctuation.) NA

\section{SECTION 3. ACTIVITIES AND USES OF THE TOXIC CHEMICAL AT THE FACILITY} (Important: Check all that apply.)

\begin{tabular}{|c|c|c|c|c|c|}
\hline 3.1 & Manufacture the toxic chemical: & 3.2 & Process the toxic chemical: & 3.3 & Otherwise use the toxic chemical: \\
\hline a. & Produce b. Import & & & & \\
\hline \multicolumn{6}{|c|}{ If produce or import: } \\
\hline c. & For on-site use/processing & a. & As a reactant & a. & As a chemical processing aid \\
\hline d. & For sale/distribution & b. & As a formulation component & b. & As a manufacturing aid \\
\hline e. & As a byproduct & c. & As an article component & c. $X$ & Ancillary or other use \\
\hline f. & As an impurity & d. & Repackaging & & \\
\hline
\end{tabular}

\begin{tabular}{l|l|l}
\hline 4.1 & 04 & (Enter two-digit code from instruction package.)
\end{tabular}

SECTION 5. QUANTITY OF THE TOXIC CHEMICAL ENTERING EACH ENVIRONMENTAL MEDIUM

\begin{tabular}{|c|c|c|c|c|c|}
\hline & & & $\begin{array}{l}\text { A. Total Release (pounds/year)(enter } \\
\text { range from instructions or estimate. }\end{array}$ & $\begin{array}{c}\text { B. Basis of estimate } \\
\text { (enter code) }\end{array}$ & c. \% From Stormwater \\
\hline 5.1 & $\begin{array}{l}\text { Fugitive or non-point } \\
\text { air emissions }\end{array}$ & NA & 0 & 0 & \multirow{3}{*}{ 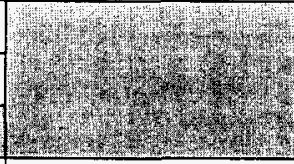 } \\
\hline 5.2 & $\begin{array}{l}\text { Stack or point } \\
\text { air emissions }\end{array}$ & NA & 0 & 0 & \\
\hline 5.3 & \multicolumn{2}{|c|}{$\begin{array}{l}\text { Discharges to receiving streams or } \\
\text { water bodies (enter one name per box) }\end{array}$} & 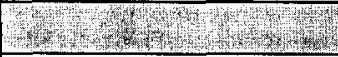 & w. & \\
\hline & \multicolumn{2}{|c|}{ Stream or Water Body Name } & & & \\
\hline 5.3.1 & \multicolumn{2}{|l|}{ COLUMBIA RIVER } & 0 & 0 & NA. \\
\hline 5.3 .2 & \multicolumn{2}{|l|}{$\mathrm{NA}$} & & & \\
\hline \multicolumn{6}{|l|}{5.3 .3} \\
\hline 5.4 .1 & $\begin{array}{l}\text { Underground Injection on-site } \\
\text { to Class I Wells }\end{array}$ & NA & NA & & \\
\hline 5.4 .2 & $\begin{array}{l}\text { Underground injection on-site } \\
\text { to Class } \|-\vee \text { Wells }\end{array}$ & NA & 0 & 0 & \\
\hline
\end{tabular}




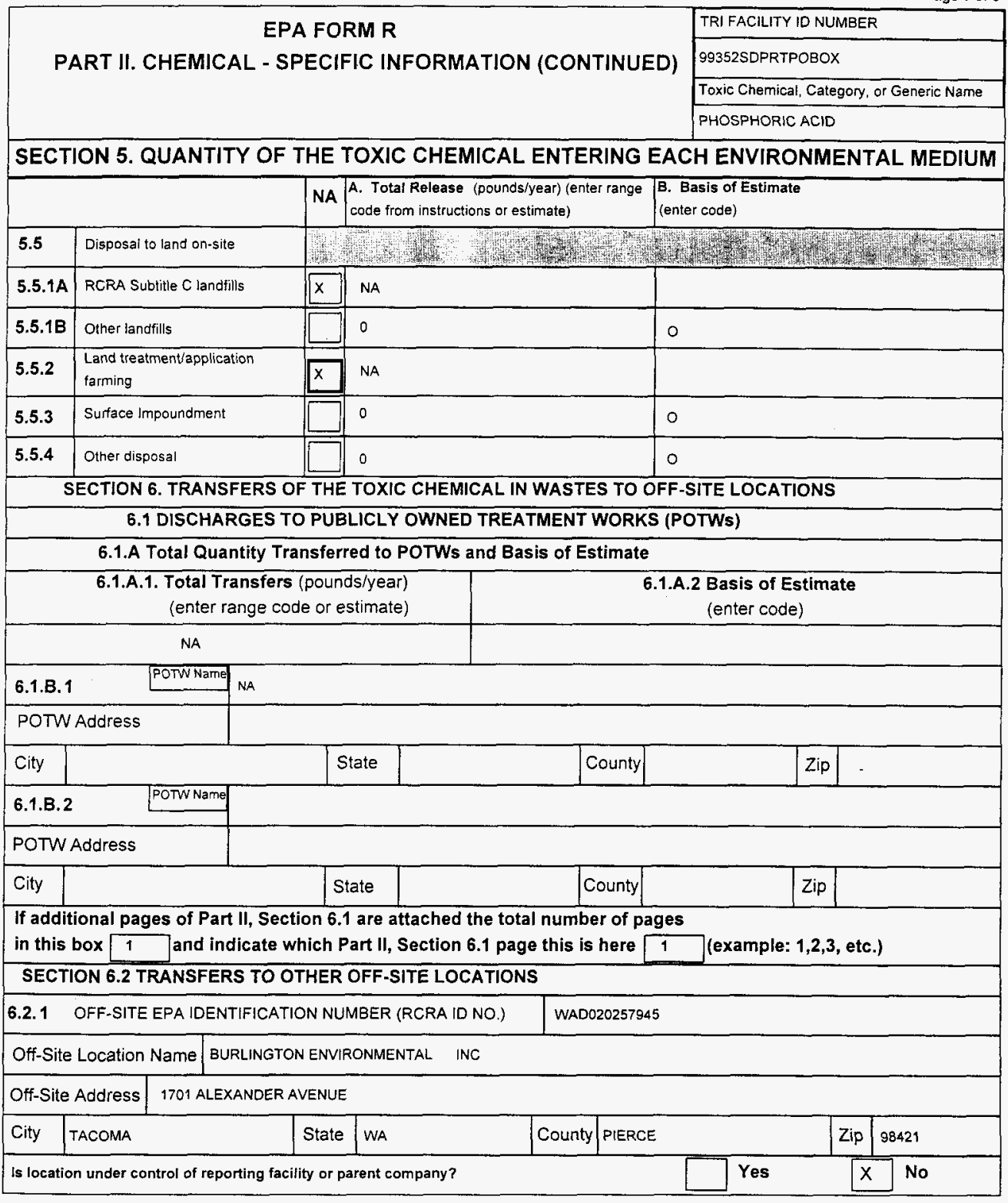




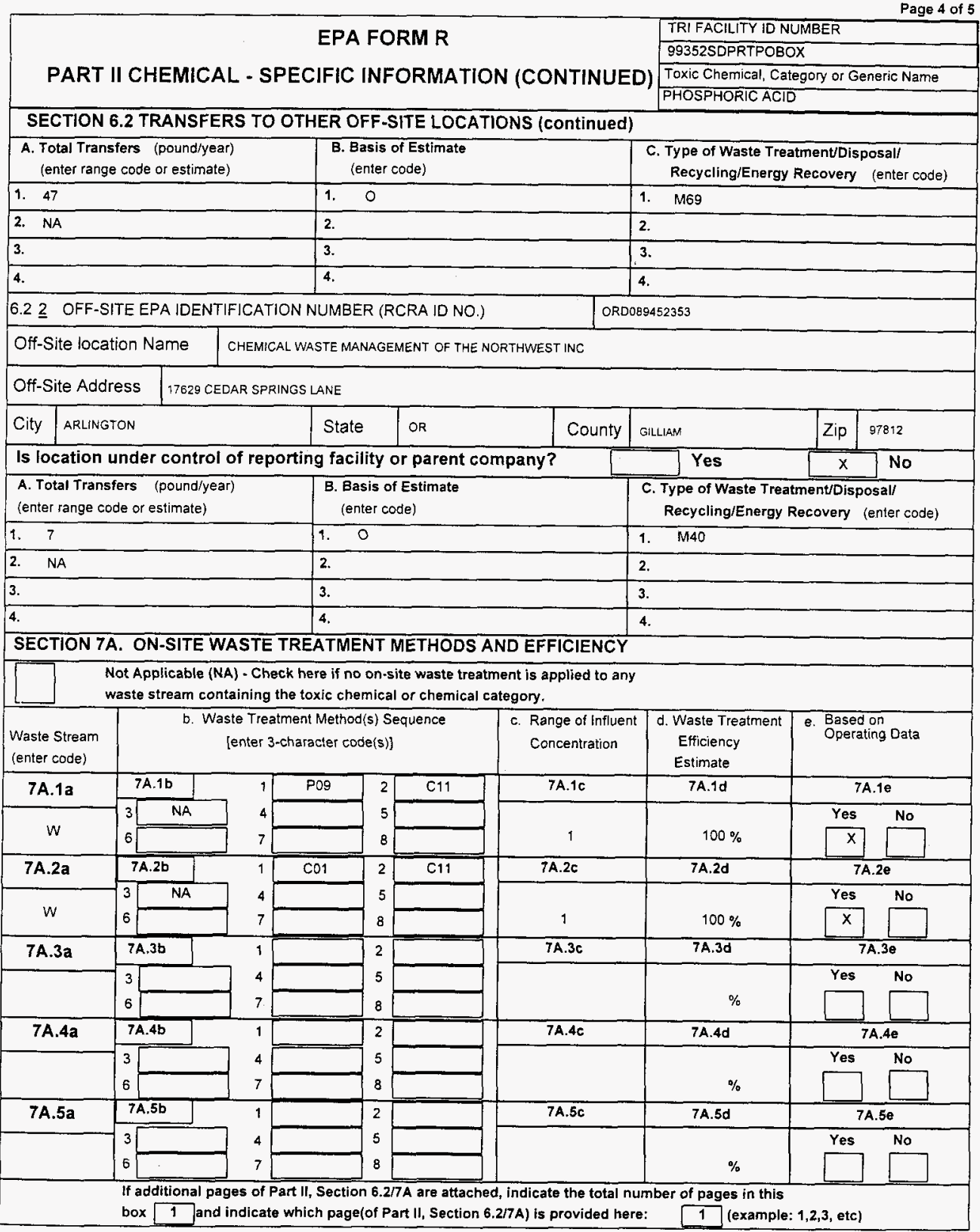


PART II. CHEMICAL-SPECIFIC INFORMATION (CONTINUED)

TRI FACILITY ID NUMBER

99352SDPRTPOBOX

Toxic Chemical, Category or Generic Name PHOSPHORIC ACID

\section{SECTION 7B. ON-SITE ENERGY RECOVERY PROCESSES}

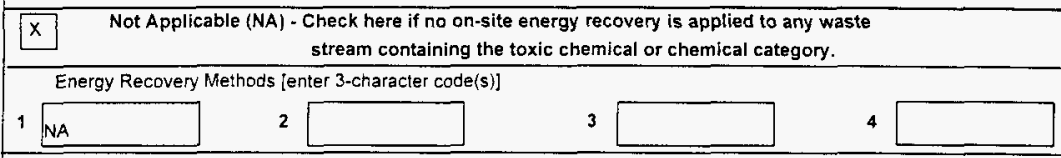

SECTION 7C. ON-SITE RECYCLING PROCESSES

$x \quad$ Not Applicable (NA) - Check here if no on-site recycling is applied to any waste stream containing the toxic chemical or chemical category.

Recycling Methods [enter 3-character code(s)]

1. NA

6.

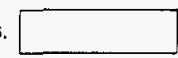

2.

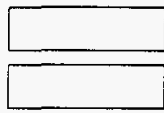

3.

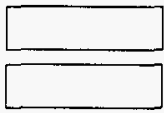

4.

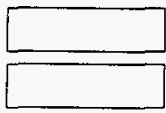

5.

10

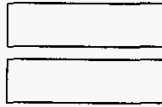

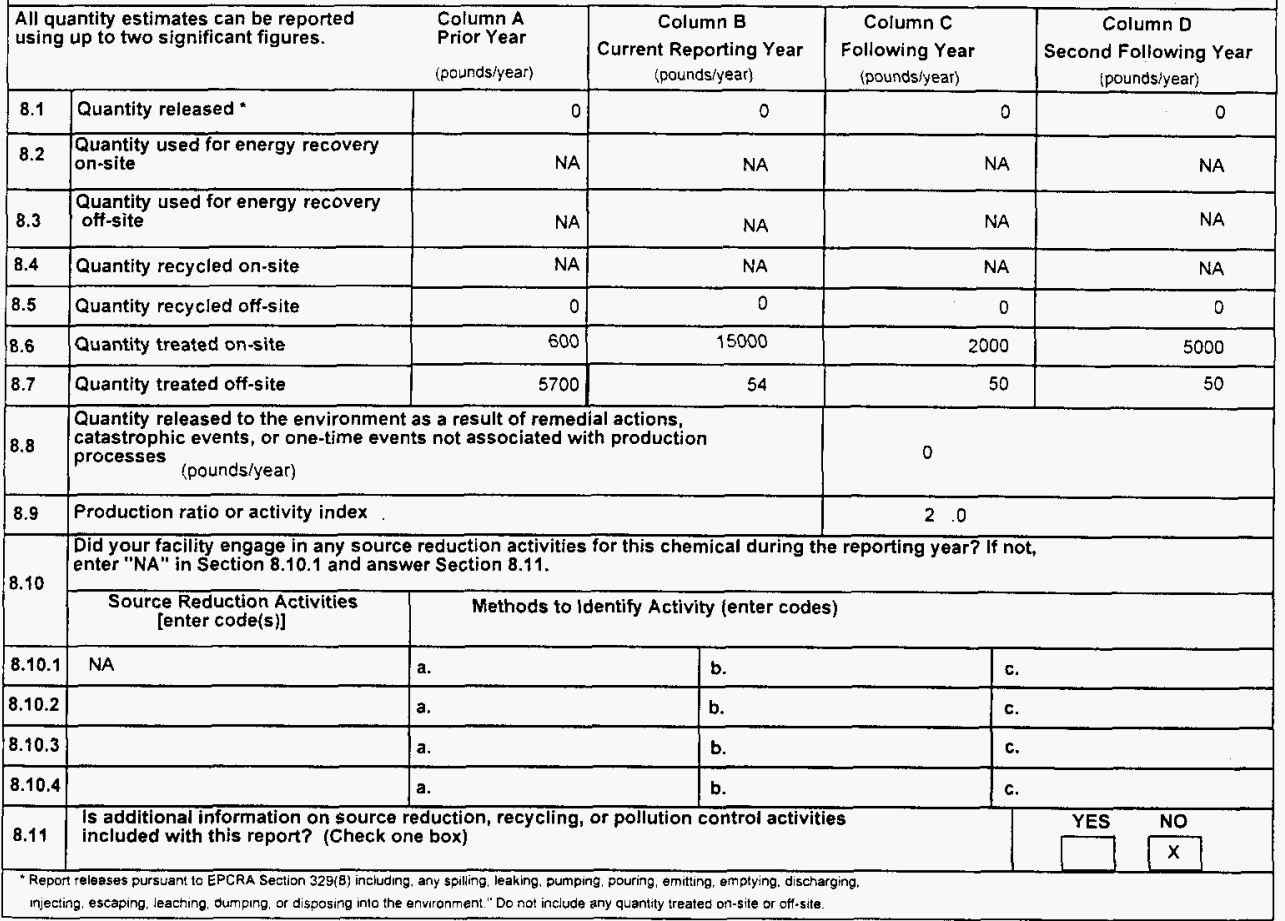




\section{HANFORD SITE TOXIC CHEMICAL RELEASE INVENTORY}

DOE-RL-98-39

\section{DISTRIBUTION}

Number

of Copies

3

2
OFF-SITE RECIPIENTS

U.S. Environmental Protection Agency

EPCRA Reporting Center

Toxic Chemical Release Inventory

Post office Box 3348

Merrifield, Virginia 22116-3348

Pesticides and Toxic Substances Branch

USEPA Region 10 (WCM-128)

1200 Sixth Avenue

Seattle, Washington 98101

Mr. Douglas R. Sherwood, Hanford Project Manager

712 Swift Boulevard, Suite 5 MS B5-01

Richland, Washington 99352

State of Washington Department of Ecology

Ms. Ide 11 Hanson

Community Right-To-know Unit

Post Office Box 47659

0lympia, Washington 98504-7585

Mr. Steven V. Moore

Hanford Project office

1315 West Fourth Avenue

Kennewick, Washington 99336-6018

Confederated Tribes of the Umatilla Indian Reservation

Mr. Jim R. Wilkinson

Board of Trustees

Post office Box 638

Pendleton, Oregon 97801 
DOE-RL-98-39

DISTRIBUTION, cont inued

Number

of Copies

OFF-SITE RECIPIENTS (continued)

1

Nez Perce Tribe

Ms. Donna Powaukee, Manager

Environmental Restoration and Waste Management Program Post Office Box 365

Lapwai, Idaho 83540

1

Wanapum Indian Band

Ms. Leanora Seetalsee

Post Office Box 878

Ephrata, Washington 98823

Yakama Indian Nation

Mr. Russel1 Jim, Manager

Environmental Restoration and Waste Management Program 2802 Main Street

Union Gap, Washington 98903

ON-SITE RECIPIENTS

11

U.S. Department of Energy, Richland Operations office

H. E. Bilson

$57-41$

R. F. Christensen

K8-50

0 . A. Farabee

R3 -79

R. A. Holten

$\mathrm{H} 0-12$

R. N. Krekel

A5- 15

J. E. Ollero

A5- 18

J. C. Peschong

S7-51

J. E. Rasmussen

A5- 15

L. D. Romine

R3 -79

W. A. Rutherford

A2 -45

E. D. Sellers

$57-41$ 
DOE-RL-98-39

DISTRIBUTION, cont inued

Number

of Copies ON-SITE RECIPIENTS (continued)

4

Fluor Daniel Hanford, Inc.

W. D. Adair

H6-21

C. G. Mattsson

N1-26

W. E. Toebe

$\mathrm{H} 6-23$

B. D. Williamson

B3 -15

3

Bechtel Hanford, Inc.

M. C. Hughes

H0-09

S. J. Ingle

H9-01

R. J. Landon

$\mathrm{HO}-02$

3

Hanford Environmental Health Foundation

J. A. Calcagni, Medical Director

$\mathrm{HI}-02$

S. M. McInturff

$\mathrm{H} 1-77$

HEHF Resource Center

$\mathrm{H} 1-79$

4

Pacific Northwest National Laboratory

G. W. McNair

P7 -79

E. A. Raney

P7 -79

DOE Public Reading Room

$\mathrm{H} 2-53$

Hanford Technical Library

P8-55

6

Waste Management Federal Services of Hanford, Inc.

T. A. Burdin

H6-20

J. 0. Skolrud

$\mathrm{H} 6-20$

A. G. Weiner

H6-21

J. A. Winterhalder

M. T. Yasdick

H6-21

D. E. Zaloudek

$\mathrm{H} 6-40$

H6-20

3

Central Files

B1-07

Document Processing Center

A3-94

EDMC

H6-08 